\title{
Identification and Classification of Atmospheric Particles Based on SEM Images Using Convolutional Neural Network with Attention Mechanism
}

\author{
Changchang Yin, ${ }^{1}$ Xuezhen Cheng ${ }^{(D},{ }^{1}$ Xilu Liu, ${ }^{1,2}$ and Meng Zhao ${ }^{1}$ \\ ${ }^{1}$ College of Electrical Engineering and Automation, Shandong University of Science and Technology, Qingdao 266590, China \\ ${ }^{2}$ Hangzhou Hikvision Digital Technology Co., Ltd., Beijing Branch, Beijing 100088, China
}

Correspondence should be addressed to Xuezhen Cheng; zhenxc6411@163.com

Received 19 June 2020; Revised 8 August 2020; Accepted 20 August 2020; Published 23 September 2020

Academic Editor: Jing $\mathrm{Na}$

Copyright (c) 2020 Changchang Yin et al. This is an open access article distributed under the Creative Commons Attribution License, which permits unrestricted use, distribution, and reproduction in any medium, provided the original work is properly cited.

\begin{abstract}
Accurate identification and classification of atmospheric particulates can provide the basis for their source apportionment. Most current research studies mainly focus on the classification of atmospheric particles based on the energy spectrum of particles, which has the problems of low accuracy and being time-consuming. It is necessary to study the classification method of atmospheric particles with higher accuracy. In this paper, a convolutional neural network (CNN) model with attention mechanism is proposed to identify and classify the scanning electron microscopy (SEM) images of atmospheric particles. First, this work established a database, Qingdao 2016-2018, for atmospheric particles classification research. This database consists of 3469 SEM images of single particulates. Secondly, by analyzing the morphological characteristics of single particle SEM images, it can be divided into four categories: fibrous particles, flocculent particles, spherical particles, and mineral particles. Thirdly, by introducing attention mechanism into convolutional neural network, an Attention-CNN model for the identification and classification of the four types of atmospheric particles based on the SEM images is established. Finally, the Attention-CNN model is trained and tested based on the SEM images database, and the results of identification and classification for four types of particles are obtained. Under the same SEM images database, the classification results from Attention-CNN are compared with those of CNN and SVM. It is found that Attention-CNN has higher classification accuracy and reduces significantly the misclassification number of particles, which shows the focusing effect of attention mechanism.
\end{abstract}

\section{Introduction}

Atmospheric particulates refer to the micro-solid or liquid matters suspending in the atmosphere. Particulate matters whose aerodynamic equivalent diameters are less than or equal to $2.5 \mu \mathrm{m}$ are called PM2.5. PM2.5 has small particle size and large surface area, and most of them are nanoscale spheres and ellipsoidal spheres. They can carry a large number of toxic and harmful substances and stay in the atmosphere for a long time. This has a great impact on physical health by penetrating deep into lung and exacerbating chronic respiratory and pulmonary diseases [1]. Also, long-term exposure to PM2.5 will increase the incidence of diseases and mortality $[2,3]$. Besides, PM2.5 in the atmosphere affects global climate: soot aerosol particles are major contributors to global warming through their absorption of solar radiation [4], and the PM2.5 response to climate change is complex and variable with different regions [5]. At present, the study of PM2.5 has become an important issue in the prevention and control of global air pollution.

Atmospheric particulates have different morphological characteristics and chemical composition. The study of single particle can well reflect its physical and chemical characteristics and plays an important role in the formation mechanism, transport process, chemical activity, and environmental impact of particulate matter. Many scholars have studied the particle size distribution, micromorphology, hygroscopicity, optical 
characteristics, element composition, and mixing state of single particles. Genga et al. [6] applied principal component analysis and hierarchical cluster analysis on PM10, whose aerodynamic equivalent diameter is less than or equal to $10 \mu \mathrm{m}$, to investigate the relationship among the chemical and morphological parameters. Based on the scanning electron microscope-energy dispersive X-ray spectroscopy (SEM-EDS), the microscopic images of the particles were processed, and the area, aspect ratio, roundness, fractal dimension, box width, box height, and perimeter of the particles were obtained through image analysis. Chithra and Shiva Nagendra [7] measured the chemical characterization of suspended particulate matter of indoor and outdoor in Chennai city during monsoon, winter, and summer seasons using ion chromatography and inductively coupled plasma optical emission spectrometry. Morphological characteristics of particles were analyzed by scanning electron microscopy (SEM) coupled with energy dispersive X-ray spectrometry, and the distribution of the chemical composition of particulate matter was obtained. Dye et al. [8] collected the fine urban aerosol $(<1 \mu \mathrm{m})$ using direct sampling of urban aerosol onto porous carbon films. Aerosol particles morphology characteristics were analyzed using transmission electron microscopy (TEM), and the fractal dimensions and diameter of particles were measured and analyzed. Akram et al. [9] investigated the morphology, microstructure, and chemical composition of a variety of particles emitted from coal-fired power plants, steel plants, and vehicle exhausts by SEM and TEM. The investigation indicated that the particles from these three sources were different in morphology, microstructure, and chemical composition. Sphere aggregates were generally the most abundant components, with silicon and aluminum as major elements. Pipal et al. [10] investigated the shapes, morphology, and elemental composition of aerosols in PM10 and PM2.5 in Agra located in north central India using SEMEDS and concluded that SEM-EDS was a convenient method to identify the sources of particulate air pollution emissions. Zhao et al. [11] investigated the morphology, chemical characteristics, and source apportionment of PM2.5 samples collected from five sites in Jiaxing. SEM and TEM were used to analyze the PM2.5 and the results showed that soot aggregates and coal-fired fly ash were the most abundant components in the samples. The results revealed that the PM2.5 concentrations in winter and spring were higher than in autumn and summer. Li and Shao [12] applied TEM and SEM to study morphologies, sizes, and compositions of aerosol particles during the fog and nonfog episode in Beijing. The number size distributions of mineral particles in fog and nonfog episodes were obtained and the results demonstrated that particles mainly included Si-rich, $\mathrm{Ca}$ rich, and S-rich. Pipal et al. [13] used medium volume air sampler to sample the PM2.5 and PM10 particles at roadside and a semirural site over Agra, India. Particle number concentrations of PM2.5 and PM10 were measured by a Grimm aerosol spectrophotometer. The morphology and elemental composition analyses of particulate matter were performed by SEM and Energy Dispersive Spectrometry (EDS), respectively. Bahadar Zeb et al. [14] studied the morphology, size, and composition of PM2.5 and PM10 by using Fourier Transform Infrared Spectroscopy and SEM with energy dispersive X-ray spectroscopy.
The abovementioned studies on the morphology of atmospheric particles mainly focus on the study of the morphology characteristics of atmospheric particles by SEM and TEM, as well as the analysis of characteristics and composition of particles by EDS. Because the background of SEM images of atmospheric particulates is complex and has many noises, it is time-consuming and labor-intensive for classification of atmospheric particles based on EDS, and an effective classification method of atmospheric particulates is needed for further investigation.

As the rising of machine learning, deep neural network, especially CNN, which has good data process capability, has gained in-depth applications in image identification and classification. In this paper, the $\mathrm{CNN}$ with attention mechanism applied to the identification and classification of SEM images of atmospheric particles is studied. The main contributions are as follows:

(1) The SEM images database of atmospheric particulates including four types of morphological characteristics is established, which contains fiber particulates, flocculent particulates, spherical particulates, and mineral particulates.

(2) In order to improve the identification and classification accuracy of $\mathrm{CNN}$, the attention mechanism is introduced into $\mathrm{CNN}$, and the Attention-CNN model for identifying and classifying SEM images of atmospheric particles is established.

(3) The Attention-CNN model is trained and tested using the SEM images database of atmospheric particles. The results indicate that the proposed Attention-CNN model outperforms CNN and SVM.

The rest of the paper is organized as follows. In Section 2, the research status related to the identification and classification of atmospheric particles is introduced. In Section 3, the processes of sample collection and SEM image acquisition of atmospheric particles are provided and the morphological characteristics of atmospheric particulates SEM images are analyzed. In Section 4, the Attention-CNN model is constructed. In Section 5, the experiment results and analysis are presented. Finally, a brief conclusion and recommendations for future work are presented in Section 6.

\section{Literature Review}

Some scholars have applied deep CNN to the study of the concentration of particulate matter and made a series of achievements. Huang and Kuo [15] combined CNN with long-term and short-term memory (LSTM) to predict PM2.5 concentration. The results showed that the forecasting accuracy of the proposed CNN-LSTM model was the best compared with the traditional machine learning methods. Chakma et al. [16] combined machine learning with natural images, classified natural images with different PM2.5 concentrations based on deep CNN, and estimated the PM2.5 concentration. Wen et al. [17] proposed a spatiotemporal convolutional long short-term memory neural network extended (C-LSTME) model for predicting PM2.5 
concentration. In the model, high-level spatiotemporal features were extracted through the combination of $\mathrm{CNN}$ and long-short-term memory neural network (LSTM-NN). The results showed that the presented model had achieved better performance. Soh et al. [18] proposed PM2.5 prediction model using a combination of multiple neural networks, including an artificial neural network, a $\mathrm{CNN}$, and a long-short-term memory to extract spatial-temporal relations. Experiments employing Taiwan and Beijing datasets showed that the proposed model achieved excellent performance. Li et al. [19] proposed a deep CNN model for exploiting the spatial influence remotely sensed PM2.5 concentration. The results demonstrated that the deep CNN model could be well applied in the field of spatially analyzing remotely sensed big data. The accuracy of the deep CNN is much higher than that of geographically weighted regression. Bo et al. [20] combined image and weather information to estimate PM2.5 indices using CNN and support vector regression (SVR) techniques. First, CNN was used to predict the PM2.5 index based on image information, and then the PM2.5 predicted by CNN and two weather features, humidity and wind speed, were combined to yield final estimated PM2.5 index using a created SVR model.

In addition, because of its powerful image learning ability, CNN is widely used in image classification and several achievements have been achieved. Ciocca et al. [21] investigated the use of CNN-based features for food recognition and retrieval, in which the architecture of CNN network was based on the Residual Network with 50 layers. Steinbrener et al. [22] used CNN to classify smaller datasets of hyperspectral images of fruits and vegetables. The proposed approach made use of the additional information from hyperspectral images to achieve improved accuracy with a limited set of training images. Deepak and Ameer [23] applied a pretrained deep network to classify brain tumor using transfer learning. The experiment showed the system recorded the best classification accuracy compared with all the related works. $\mathrm{Wu}$ et al. [24] studied different CNN algorithms for the classification of face skin diseases, which was based on the clinical images. The results demonstrated that CNNs have the ability to recognize facial skin diseases and the models using transfer learning achieved a higher average precision. Zhou et al. [25] proposed a CNN model based radiomics approach on shear-wave elastography to classify malignant and benign breast tumors. The proposed method was designed to directly extract features from the dataset without the prerequisite of segmentation and manual operation. Compared with the existing methods, the method had superior performances. In [26], a mathematical model based on transfer learning is used, in which a CNN architecture, VGG-16 trained on ImageNet dataset, was used as a feature extractor. Experimental results demonstrated that an excellent accuracy was achieved. In [27-32], hyperspectral images were classified based on CNN and its improved methods, and the superiority of the corresponding methods was verified through experiments. Ma et al. [33] presented a hybrid $\mathrm{CNN}$ for classification of pollution levels based on images with PM2.5 values. The method achieved excellent performance on classification of air pollution levels from a single captured image.

Due to the focusing effect of the attention mechanism, it is added to the CNN to assign different weights to different information, filter out unimportant information, and enable the model to focus on key information areas of the image, thereby improving the identification and classification accuracy of the CNN model. Li et al. [34] established a largescale attention-based glaucoma (LAG) database and proposed an attention-based CNN for glaucoma detection, called AG-CNN. The experiment results from testing over LAG database and another public glaucoma database showed that the AG-CNN significantly advanced the state of the art in glaucoma detection. Karthik et al. [35] exploited the features learned by $\mathrm{CNN}$ at various processing hierarchies using the attention mechanism and achieved an overall accuracy of $98 \%$ on the validation sets in the 5 -fold crossvalidation. Li et al. [36] proposed a deep learning-based method for urban PM2.5 concentration prediction, which comprised a one-dimensional CNN, LSTM, and attentionbased network. The results indicate that the method is capable of dealing with PM2.5 concentration prediction and outperforms six baseline methods. Song et al. [37] presented a highly discriminative network, namely, attention branch based convolutional neural network (ABCNN), to distinguish the differences between leaves features. The ABCNN was tested on special dataset of LeafSnap with highly similar tree leaves and achieved $91.43 \%$ classification accuracy, which is higher than previous methods.

In the above-cited works for the study of atmospheric particulates, obtaining SEM images is the basic method. SEM images of atmospheric particulates can be used to analyze the morphology of particles, which are widely used in the study of atmospheric particles. In addition, $\mathrm{CNN}$ has strong image learning and classification ability, and it is mainly used to analyze and predict the concentration of particulates in the study of atmospheric particulates. There are few researches on the application of deep CNN in the classification of atmospheric particulates based on SEM images. In $[16,33,36]$, CNN and its improved algorithms were used to classify and study the natural images of atmospheric particulates, mainly solving the problem of particulate matter concentration and pollution level. Morphological characteristics of atmospheric particulates SEM images, affecting particulate source apportionment, should be further studied. In this paper, the SEM images of atmospheric particulates are discussed based on the morphological characteristics of PM2.5. The CNN and attention mechanism are applied to identify the SEM images of atmospheric particles and realize the classification of four types of particles.

\section{Sample Collection and Preparation}

3.1. Sampling Sites of Atmospheric Particles. Seven environmental monitoring sites in Qingdao, China, are selected to collect atmospheric particulates, which are Qingdao Port, Sino-German Eco-Park, Citizen Culture Square, Hengyuan Thermal Power Plant, Huangdao Power Plant, North of 
Xinjiekou, and Shandong University of Science and Technology (SDUST). Information of the seven monitoring sites includes name, sampling height, functional area, and geographical location, which are shown in Table 1. The sampling times include four seasons and three years, which are the summer of 2016 (July 3-22), the autumn of 2016(October 6-19), the spring of 2017 (April 4-20), the autumn of 2017 (September 5-21), the spring of 2018 (April 6-19), and the winter of 2018 (November 8-21). Each sampling period is between 2 and 5 hours.

3.2. Sampling Filter Membranes for Atmospheric Particulates. Generally, there are three kinds of sampling filter membranes for atmospheric particulates: quartz fiber filter membrane, cellulose acetate filter membrane, and polycarbonate filter membrane. Under the scanning electron microscope, the morphology of these three filter membranes can be observed from the microscopic level.

As shown in Figure 1(a), the quartz fiber filter membrane shows complicated fibrous particles in the SEM. Because of the large thickness of quartz fiber filter membrane, tiny particulates are easy to be embedded in the fiber gap, which makes it inconvenient for operators to obtain images. However, the membrane has high temperature resistance, corrosion resistance, good air permeability, and high flow rate sampling.

The morphology of cellulose acetate filter membrane in the SEM presents rough pore morphology, as shown in Figure 1(b). The particulates are embedded in the holes or gaps. The cellulose acetate filter membrane has the advantages of being thinner than the quartz fiber filter membrane, good air permeability, relatively complete adsorbed particulates, and relatively taking less time to find particulate. However, due to the influence of membrane fiber, it is easy to occlude particles.

The microscopic morphology of the polycarbonate filter membrane is very clear in the SEM, and the surface presents a smooth microporous shape, as shown in Figure 1(c). SEM image shows that the particulates adhere to the surface of the membrane and the holes are tiny. As background, polycarbonate filter membrane is very suitable for analyzing of PM2.5 using image processing. Polycarbonate filtration membrane also has disadvantages. Due to the small holes and poor permeability, smaller air flow and longer sampling time are required when collecting samples.

The three filter membranes have their own advantages and disadvantages. In this paper, deep learning method is adopted to identify atmospheric particulates. SEM images with different backgrounds are more conducive to the applicability of the network model. Therefore, all three filter membranes are used for sample collection.

3.3. Preparation of Samples. The fine particulate sampler and medium flow PM2.5 sampler are used for sampling PM2.5. Sampling filters are quartz fiber filters, cellulose acetate filters with $0.8 \mu \mathrm{m}$ pore size, and polycarbonate filters with $0.1 \mu \mathrm{m}$ pore size. The sampling flow of particles was calibrated before and after sampling, and blank filter membrane was left on-site for sampling to analyze whether the sampling process was contaminated.

In order to obtain the SEM images of PM2.5, three steps are needed. First, make observation sample: an area of size $5 \mathrm{~mm} \times 10 \mathrm{~mm}$ was cut on the filter randomly and adhered to SEM sample piles made of aluminium by conductive adhesives. Second, coat the observation sample with platinum: samples were placed in the spray tank and coated with platinum on the surface by ion sputtering under condition of vacuum. Third, sample piles were placed in scanning electron microscopy (US FEI Nova Nano SEM 450), and the SEM images of PM2.5 were obtained under high vacuum mode with an accelerating voltage of $20 \mathrm{KV}$ and magnification of 20,000 times.

\subsection{Morphological Characteristics of Atmospheric Particulates} SEM Images. Based on previous research by our group [38], the observation and analysis of a large number of PM2.5 SEM images, it is found that there are four types of particulates with obvious morphological characteristics, namely, fibrous particulates, flocculent particulates, spherical particulates, and mineral particulates. The SEM images of the four types of atmospheric particulates are shown in Figure 2.

The morphological characteristics of fibrous particulates are obvious and their surface is smooth and long. They are generally derived from minerals, plant fibers, and artificial fibers dispersed in the air. Fibrous particulates mostly belong to primary aerosol, which are not transformed by secondary chemical reaction in the atmosphere.

Flocculent particulates are formed by a large number of $30-50 \mathrm{~nm}$ round particles gathered together, mainly from the fine particles emitted by motor vehicles adsorbing other elements in the air through secondary transformation and condensation. Their morphological characteristics are chain and cluster in general, which are easy to distinguish [39].

Spherical particulates are generally known as coalfired fly ash. Due to their smooth spherical characteristics, they are easily identified in atmospheric particles. The main sources of spherical particulates are coal combustion, biomass combustion, or garbage incineration, the formation mechanism of which is the melting of solid fly ash at high temperature. The size of spherical particles is micron generally, and their surface can also adsorb other ultrafine particles or secondary particles.

Mineral particulates generally come from dust and products of secondary atmospheric chemical reactions. Fugitive dust mainly includes road fugitive dust, soil fugitive dust, building fugitive dust, and industrial fugitive dust. Their morphological characteristics are diverse and generally manifested as mineral form or regular block, which are difficult to distinguish. Mineral particulates come from fine particulate matter in windblown dust on the surface of Earth. Regular mineral particulates generally come from chemical reactions in the atmosphere. 
TABLE 1: Information of sampling sites.

\begin{tabular}{lcccc}
\hline No. & Sampling site & Sampling height $(\mathrm{m})$ & Functional area & Geographical location \\
\hline 1 & Qingdao Port & 5 & Industrial area & $120^{\circ} 19^{\prime} \mathrm{E}, 36^{\circ} 04^{\prime} \mathrm{N}$ \\
2 & Sino-German Eco-Park & 10 & Residential area & $120^{\circ} 13^{\prime} \mathrm{E}, 36^{\circ} 07^{\prime} \mathrm{N}$ \\
3 & Citizen Culture Square & 5 & Marine ecological zone & $120^{\circ} 20^{\prime} \mathrm{E}, 35^{\circ} 97^{\prime} \mathrm{N}$ \\
4 & Hengyuan Thermal Power Plant & 5 & Industrial area & $120^{\circ} 13^{\prime} \mathrm{E}, 36^{\circ} 01^{\prime} \mathrm{N}$ \\
5 & Huangdao Power Plant & 10 & Industrial area & $120^{\circ} 22^{\prime} \mathrm{E}, 36^{\circ} 05^{\prime} \mathrm{N}$ \\
6 & North of Xinjiekou & 10 & Urban-rural area & $120^{\circ} 16^{\prime} \mathrm{E}, 36^{\circ} 03^{\prime} \mathrm{N}$ \\
7 & SDUST & 10 & Cultural and educational area & $120^{\circ} 12^{\prime} \mathrm{E}, 36^{\circ} 00^{\prime} \mathrm{N}$ \\
\hline
\end{tabular}

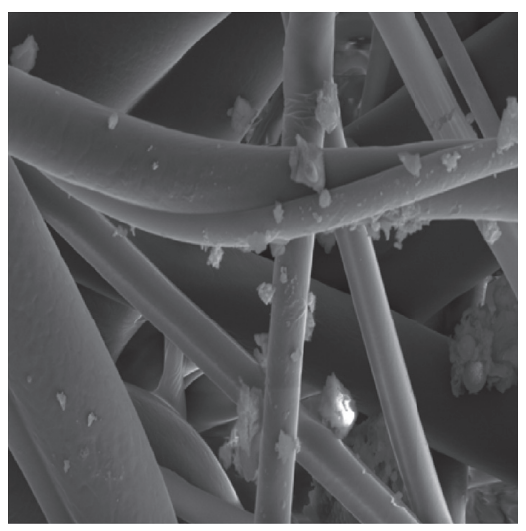

(a)

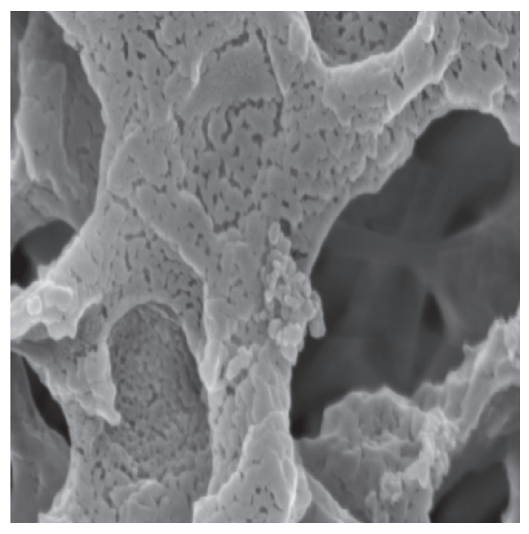

(b)

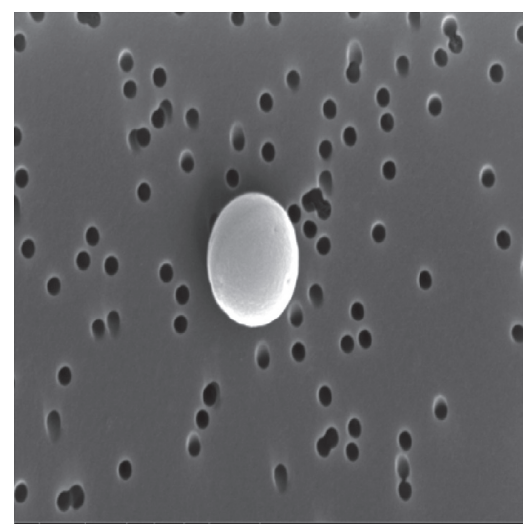

(c)

FIGURE 1: Samples of three filter membranes: (a) quartz fiber filter membrane; (b) cellulose acetate filter membrane; (c) polycarbonate filter film membrane.

\section{Convolutional Neural Network with Attention Mechanism}

4.1. Convolutional Neural Network. Convolutional neural network is one of the common frameworks of deep learning, which has been widely used because of its unique advantages in image processing. For image processing, convolution is applied to the digital image spatial domain to directly calculate the original pixel space, that is, to obtain important features in the image by multi-convolution kernel operation. At the same time, the back propagation algorithm based on gradient descent is used to train the weights of the network, which has good robustness to translation, scaling, tilt, and so on, and finally the accurate identification and classification of the image is achieved.

CNN applied to image identification and classification mainly includes five parts: input layer, convolution layer, pooling layer, full connection layer, and output layer, as shown in Figure 3. Among them, the convolution layer uses convolution kernel to obtain image features and realize filtering and strengthening of image features. The pooling layer reduces the feature dimension of convolution layer by downsampling, compresses the amount of data and parameters, and reduces overfitting. The full connection layer connects all input nodes in series into vectors and outputs image features.

4.2. Attention Mechanism. Attention mechanism is a kind of model similar to the attention function of human. It draws on the principle that people will focus their attention on a specific location at a specific time and reduce their attention to other locations. The function of attention mechanism can be interpreted as screening important information from multiple information, focusing on important information, and ignoring unimportant information. Attention mechanism uses the method of assigning different weights to the input features of the model to focus on important information. The focus process of important information is the calculation process of weight coefficients. The higher the importance of information, the greater the weight coefficients assigned.

The principle of attention mechanism [40] is shown in Figure 4 , in which $X=\left(x_{1}, x_{2}, \ldots, x_{n}\right)$ represents the input at time $t, H=\left(h_{1}, h_{2}, \ldots, h_{n}\right)$ indicates the hidden state information in the input sequence, and each $h_{i}(i=1,2, \ldots, n)$ contains the information learned from the input sequence by CNN. The information in $h_{i}$ mainly focuses on the input data around the $i$ th input sequence, and $y_{t}$ is the output at time $t$, which can be expressed as

$$
y_{t}=g\left(s_{t}, c_{t}\right) \text {, }
$$

where $g(\cdot)$ is CNN, $s_{t}$ is hidden output-state at time $t$, and $c_{t}$ represents context state vector obtained through learning. The calculation of $s_{t}$ can be expressed as follows:

$$
s_{t}=g\left(s_{t-1}, y_{t-1}, c_{t}\right) \text {. }
$$

The calculation formula of $c_{t}$ is as follows: 

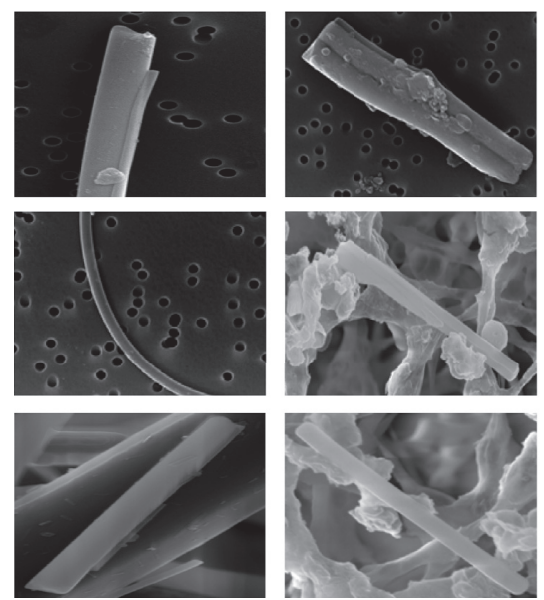

(a)
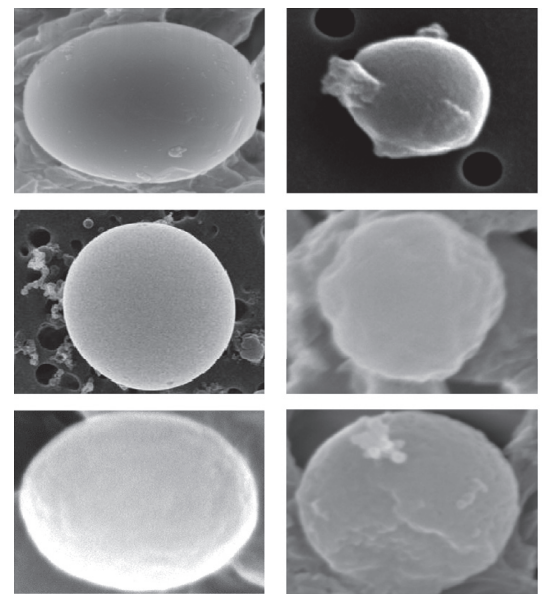

(c)
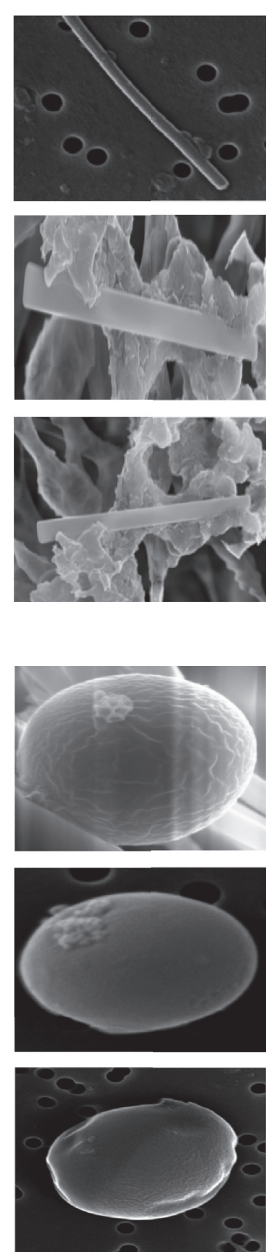
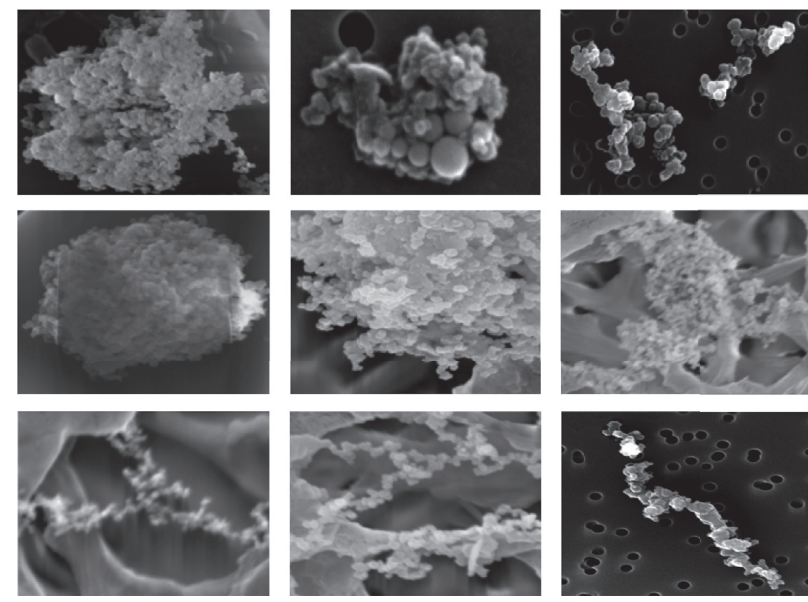

(b)
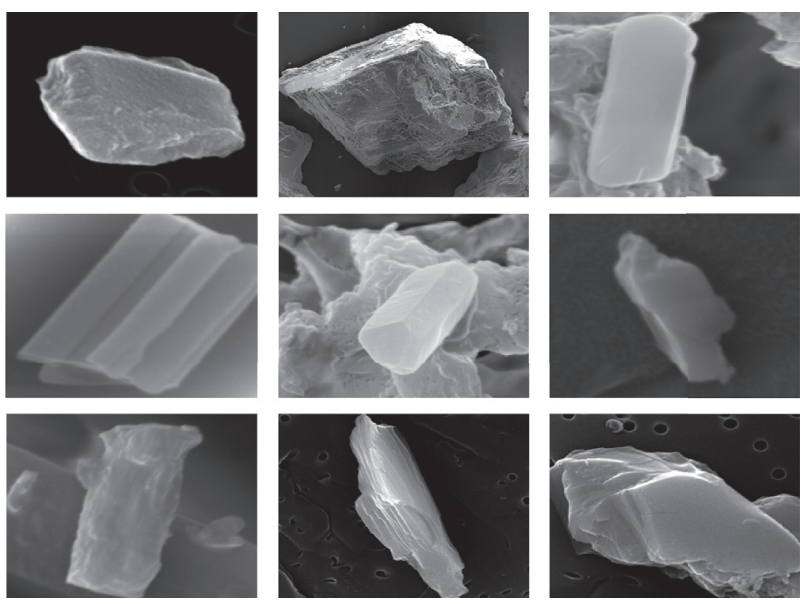

(d)

FIGURE 2: SEM images of the four types of particulates: (a) fibrous particulates; (b) flocculent particles; (c) spherical particulates; (d) mineral particulates.

$$
c_{t}=\sum_{i=1}^{n} a_{t i} h_{i},
$$

where $a_{t i}$ is the attention parameter, which corresponds to the weight of $h_{i}$; that is, its calculation formula is as follows:

$$
a_{t i}=\frac{\exp \left(e_{t i}\right)}{\sum_{j=1}^{n} \exp \left(e_{t j}\right)},
$$

where $e_{t i}$ is an alignment model, which evaluates the matching degree between the output at time $t$ and the information at the ith input. It can be calculated by learning the hidden state $s_{t-1}$ and the $h_{i}$. The calculation formula is as follows:

$$
e_{t i}=\tanh \left(W_{a} s_{t-1}+U_{a} h_{i}+b_{a}\right)
$$

where $\tanh (\cdot)$ is the activation function, $W_{a}$ and $U_{a}$ are the weight parameters, and $b_{a}$ is the bias parameter.

From the above, it can be seen that the attention mechanism can improve the learning of the influence relationship of different model states by training the influence weight of each input $x_{i}$ on the output $y_{i}$, so that the model can focus on the information that the input data has a greater impact on the output and improve the accuracy of the output $y_{i}$.

4.3. Attention-CNN Model. By introducing attention mechanism into CNN, the network can adaptively allocate weight information for the data of the whole network through the output of last moment and the input of current moment. The key information of the image can be focused on, so as to improve the accuracy and adaptability of the classification.

In this paper, atmospheric particulates are classified by identifying the SEM images. It is necessary to identify the morphology characteristics of particulates in the SEM images. However, SEM images are mainly composed of particulates and filter membrane which is equivalent to the background. If attention mechanism is integrated into CNN to recognize particles in SEM images, attention to features of particle shape can be focused on, and that to the background and other non-particle shape features can be reduced. The identification accuracy of particles can be improved, and then the classification accuracy is improved. 


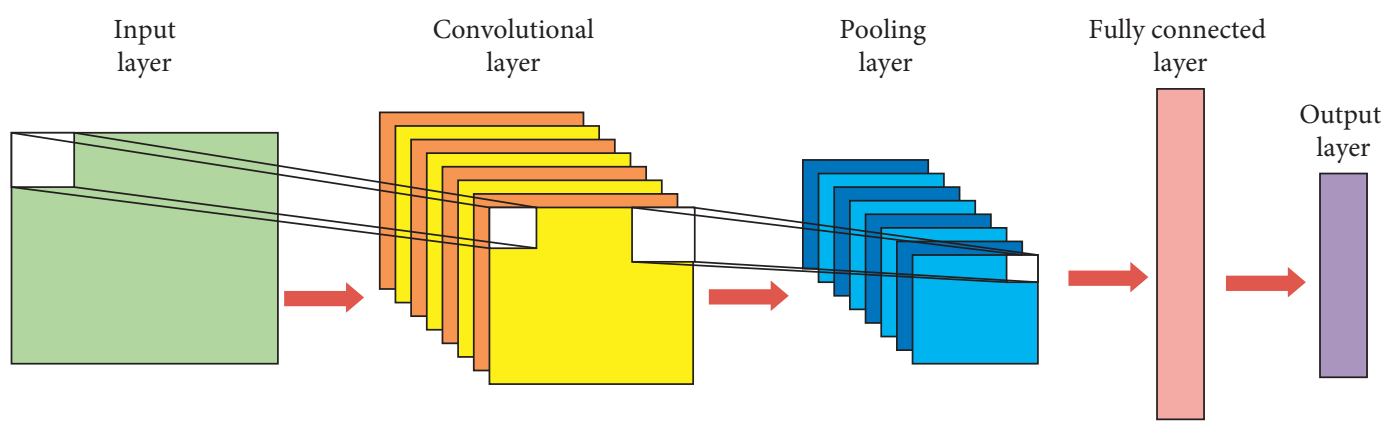

Figure 3: The architecture of CNN.

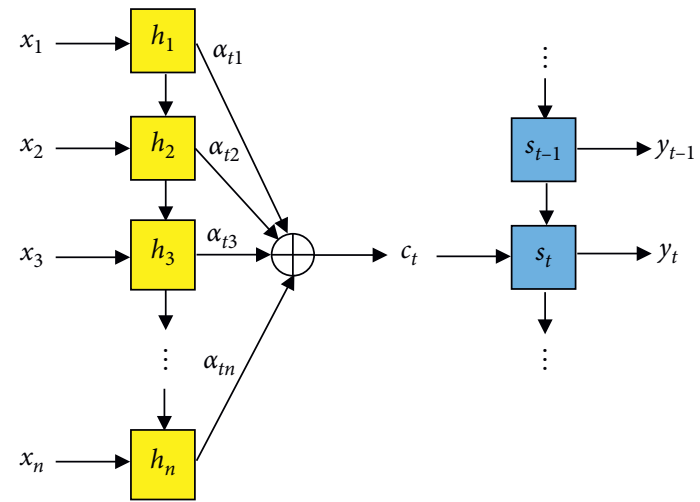

Figure 4: The principle of attention mechanism.

Based on this, Attention-CNN model is established by integrating attention mechanism into CNN. The AttentionCNN is used to identify and classify the SEM images of atmospheric particles, and its structure is shown in Figure 5. Attention-CNN model includes input layer, convolution layer, attention layer, full connection layer, and output layer.

Since the input is the SEM image of four types of particles, the input layer is four nodes, that is, the labeled SEM images of four types particles. The convolution layer is set as four layers, in which the convolution kernels of each layer are $8 \times 3 \times 3$, $16 \times 5 \times 5,32 \times 3 \times 3$, and $32 \times 3 \times 3$ successively, and attention layer is added after the convolution layer of each layer to realize the weight distribution. Pooling layer is added to the first and last convolution layer. The output of the convolution layer is the input of the full connection layer, and the node number of full connection layer is set as 64 . The output layer divides the particles into 4 categories, and the number of output layer node is 4 .

In this paper, the TensorFlow model is used to construct the Attention-CNN model. The main parameters of the model are analyzed as follows.

4.3.1. Activation Function. A nonlinear function can be introduced between the layers of the neural network by setting the activation function, and the output of the node is no longer linear, so it can fit various curves and enhance the expressive ability of the network. In Attention-CNN model, two activation functions, Relu (rectified linear unit) and softmax function, are used in the hidden layer and output layer, respectively.

Relu can solve gradient dispersion in the process of parameter transfer. When Relu function is greater than 0 , its derivative is 1 . It is simple to find the gradient, which can improve the convergence rate of gradient descent greatly. The expression of Relu function is as follows:

$$
\text { Relu }=\max (0, x) .
$$

Softmax maps the output of multiple neurons to $(0,1)$ and performs multiple classifications. Assuming there is an input array, $i$ represents the $i$ th element; the softmax value of this element is calculated by

$$
S_{i}=\frac{e^{i}}{\sum_{j=1}^{n} e^{j}},
$$

where $n$ represents all input elements.

4.3.2. Optimization Strategy. In the whole training process of deep learning model, it is necessary to use optimization algorithm to iterate the model parameters and reduce the loss function value. Some parameter optimization algorithms have been proposed and applied in related fields [41-44]. Because the Adam optimization algorithm makes full use of the first-order moment mean and second-order moment mean of the gradient to calculate the adaptive learning rate parameters, and the momentum factor is included in the updating process [45], in this paper, the Adam algorithm is chosen as the optimization strategy in Attention-CNN. The calculation process of Adam is as follows:

$$
\begin{aligned}
m_{t} & =\beta_{1} m_{t-1}+\left(1-\beta_{1}\right) g_{t}, \\
v_{t} & =\beta_{2} v_{t-1}+\left(1-\beta_{2}\right) g_{t}^{2}, \\
\widehat{m}_{t} & =\frac{m_{t}}{1-\beta_{1}^{t}}, \\
\widehat{v}_{t} & =\frac{v_{t}}{1-\beta_{2}^{t}}, \\
W_{t+1} & =W_{t}-\frac{\eta}{\sqrt{\widehat{v}_{t}}+\varepsilon} \widehat{m}_{t},
\end{aligned}
$$




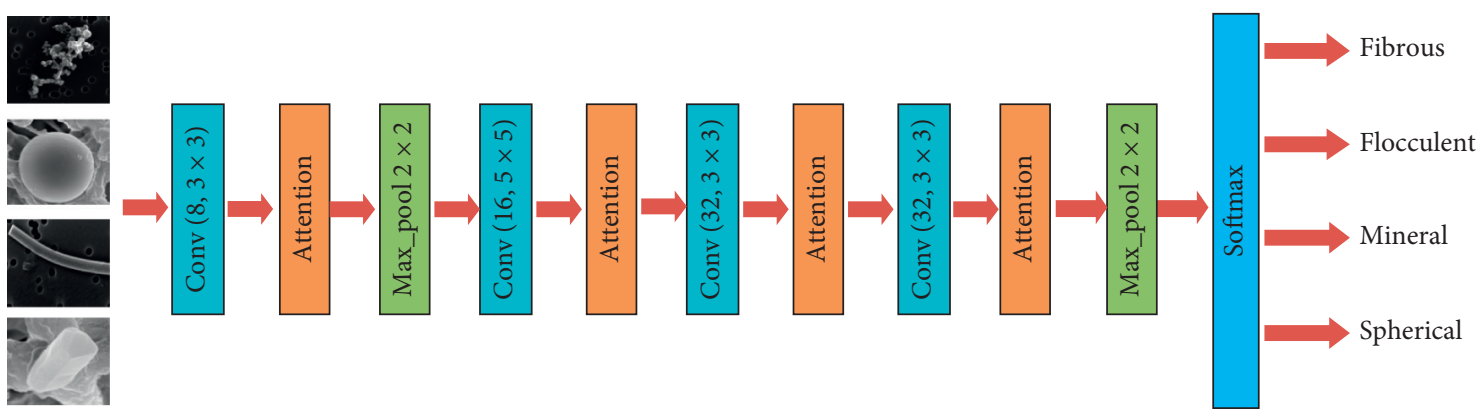

Figure 5: The structure of Attention-CNN model.

where $m_{t}$ is first-order moment estimate, $v_{t}$ is second-order momentum term, $\beta_{1}, \beta_{2}$ are dynamic values, $g_{t}$ is the gradient of the cost function after $t$ iterations, $\widehat{m}_{t}$ is correction value of first moment, $\widehat{v}_{t}$ is correction value of second moment, $W_{t}$ is the parameters of the $t$ iteration model, and $\varepsilon$ is a small number that can avoid the zero denominator.

4.3.3. Loss Function. Loss function is used in the training of neural network to evaluate the difference between the predicted value and the real value and is a standard to test the performance of the model. In this paper, cross-entropy cost function is selected as loss function in Attention-CNN, which can be expressed as

$$
C=-\frac{1}{n} \sum_{x}[y \ln a+(1-y) \ln (1-a)],
$$

where $x$ is the sample, $n$ is the sum of the samples, $a$ is the output value, and $y$ is the actual value.

The gradient of $w$ is calculated as follows:

$$
\frac{\partial C}{\partial w_{j}}=\frac{1}{n} \sum_{x} x_{j}(\sigma(z)-y),
$$

where $\sigma(z)-y$ is the error between the output and the actual value.

\section{Experiment and Results Analysis}

5.1. Database. According to the PM2.5 data collected by three kinds of sampling filter membranes from seven environmental monitoring sites in Qingdao, SEM images of atmospheric particulates were obtained, which contained four types of particulates: fibrous particulates, flocculent particulates, spherical particulates, and mineral particulates.

The collected SEM images were transmitted to the computer, and a total of 525 SEM images were obtained. Since the SEM images usually have the information bar of images, which include the shooting time, magnification, scale, and other information of the images, before image processing, it is necessary to cut the SEM images so as to remove the image information bar to avoid affecting the processing of SEM images. By cropping and rotating processing, 3469 single-particle SEM images of four types of atmospheric particles are obtained and the database is set, that is, Qingdao 2016-2018. According to the Attention-
CNN model established, 2428 SEM images in the database are used as training samples, and the remaining 1041 images are used as test samples. The sample number of four types of atmospheric particles and the number of training and testing samples in Attention-CNN are shown in Table 2.

5.2. Results and Discussion. The Attention-CNN model in Section 4.3 is trained and tested based on the SEM images database of four types of atmospheric particles. At the same time, in order to verify the performance of Attention-CNN model, the CNN and SVM which are commonly used in image classification are also applied to the identification and classification of SEM images of atmospheric particles. The structure of CNN is the same as that of the Attention-CNN model, but there is no attention layer. The three models were trained and tested using the same SEM images database. In Attention-CNN and CNN, the Relu function is selected as the activation function of convolution layer, softmax function as the activation function of output layer, Adam optimizer as the network optimization strategy, and the cross-entropy cost function as the loss function, the training time is set as 200 iterations, and the batch size is set as 128 .

After Attention-CNN, CNN, and SVM are trained and tested, the corresponding results are obtained. The accuracy and loss curves of training samples and test samples of Attention-CNN are obtained, as shown in Figure 6. Figure 7 shows the classification results of four types of particles obtained by three models.

It can be seen from Figure 6(a) that the classification accuracy of training samples gradually increases and tends to be stable with the number of iterations increasing. The classification accuracy of the test samples increases rapidly at the initial stage of the iteration. After the initial fluctuations, with the number of times increasing, the accuracy of the test samples is close to the accuracy of the training samples and is basically stable. As can be seen from Figure 6(b), similar to the changes in the accuracy of the training samples and the test samples, the loss function of the training samples and test samples decreases rapidly with the number of iterations increasing and finally tends to be stable. The changing of classification accuracy and loss function of Attention-CNN model with the number of iterations increasing shows that the design of Attention-CNN model is reasonable.

As shown in Figure 7, the Attention-CNN model is superior to the other two models in the classification of four 
TABLe 2: Sample number of atmospheric particles and the number of training and testing samples in Attention-CNN.

\begin{tabular}{lccc}
\hline Class name & Samples (3469) & Training samples (2428) & Test samples (1041) \\
\hline Fibrous & 999 & 700 & 299 \\
Flocculent & 1001 & 701 & 300 \\
Spherical & 763 & 540 & 223 \\
Mineral & 706 & 487 & 219 \\
\hline
\end{tabular}

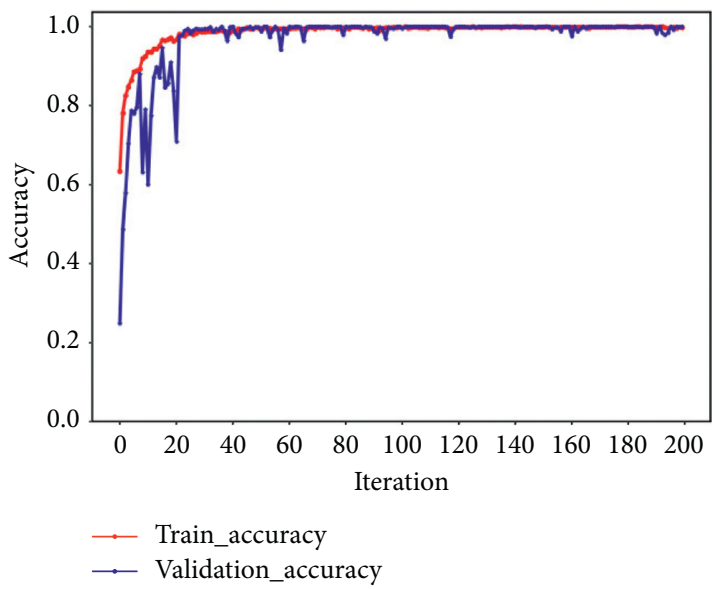

(a)

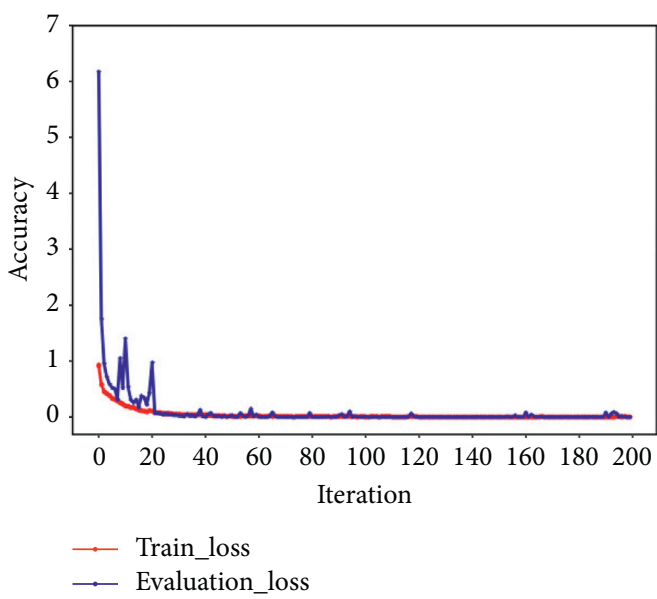

(b)

Figure 6: Accuracy and loss curve of training samples and test samples of Attention-CNN. (a) Accuracy curve; (b) loss curve.

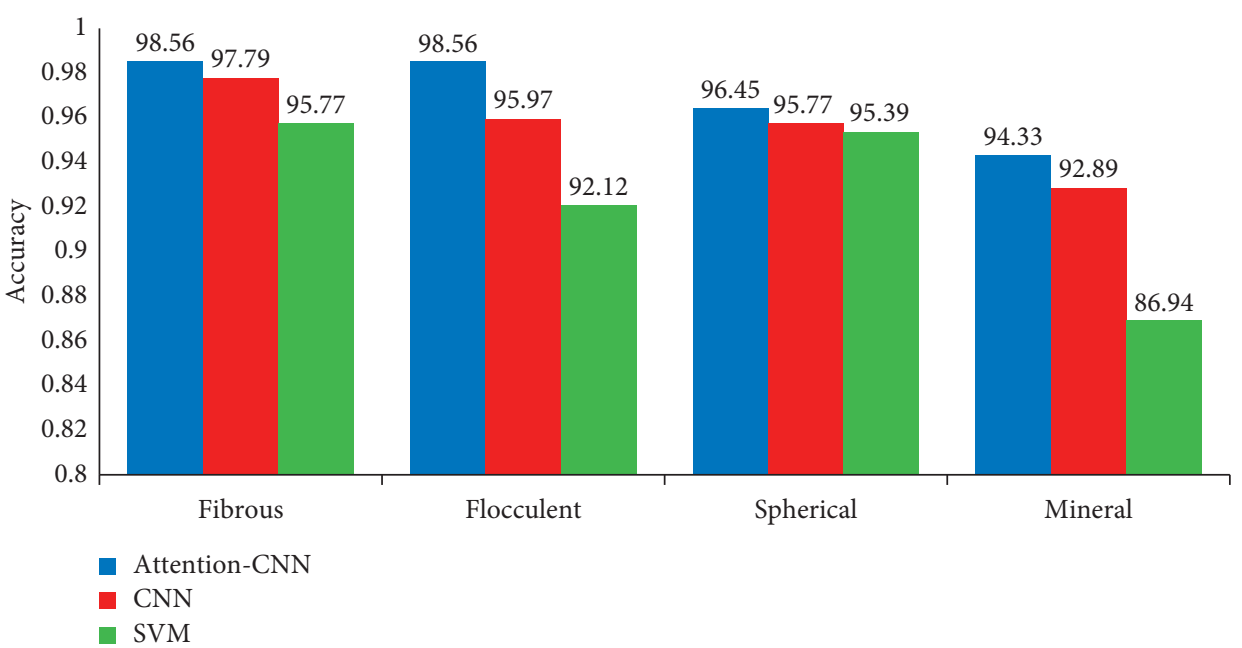

FIGURE 7: Histogram of classification accuracy of Attention-CNN, CNN, and SVM.

types of atmospheric particles. Classification accuracy of fibrous and flocculent reaches $98.56 \%$, and classification accuracy of mineral is the least, which is $94.33 \%$. The classification accuracy of the four types of particles using Attention-CNN has been improved by $0.77 \%, 2.59 \%, 0.68 \%$, and $1.44 \%$, respectively, compared with using CNN and increased by $2.79 \%, 6.44 \%, 1.06 \%$, and $7.39 \%$, respectively, compared with using SVM. This shows that the AttentionCNN model can classify the SEM images of atmospheric particles effectively.
In addition, in order to investigate the advantages of Attention-CNN over CNN and SVM in identifying and classifying SEM images of atmospheric particulates, the performances of three models are compared by the confusion matrix, as shown in Figure 8.

According to Figure 8, the following conclusions can be obtained:

(1) For fibrous particles, Attention-CNN, CNN, and SVM all have the problem of misclassifying them as 


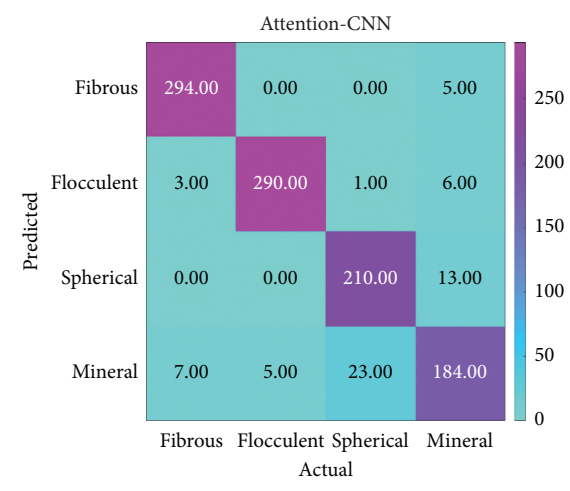

(a)

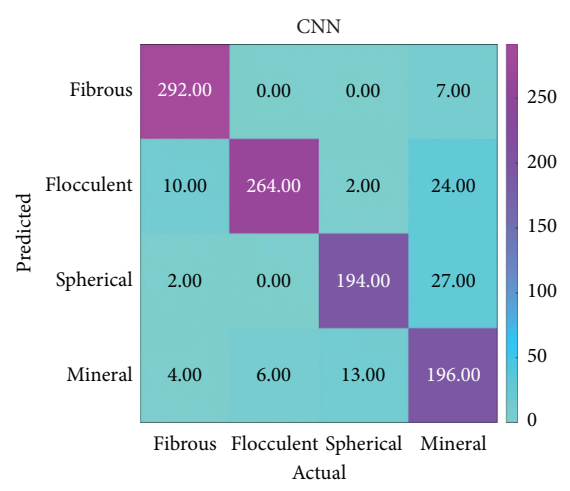

(b)

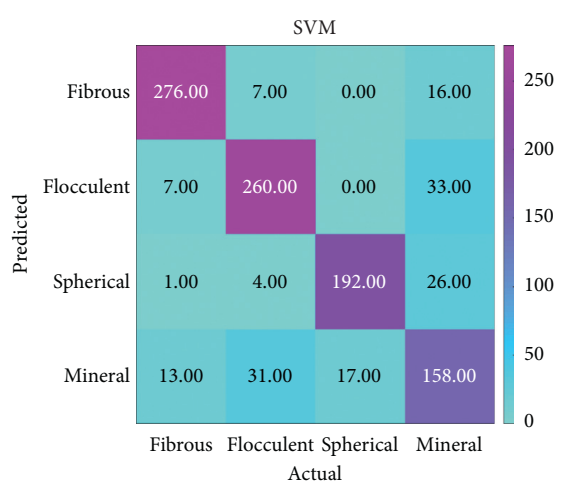

(c)

FIgure 8: Confusion matrix for classification using Attention-CNN, CNN, and SVM.

mineral particles. However, Attention-CNN misclassifying fibrous particulates as mineral particles is in the least number, followed by CNN and SVM, and SVM also has the problem of misclassifying them as flocculent particulates.

(2) For flocculent particulates, Attention-CNN and $\mathrm{CNN}$ have classified them into the other three types of particulates mistakenly. SVM misclassifies them as fiber particulates and mineral particulates. But the total number of particulates misclassified using Attention-CNN is the least, followed by $\mathrm{CNN}$ and SVM. Among them, the number of mineral particles that have been misclassified is the largest. The misclassified number of Attention-CNN, CNN, and SVM is 6, 24, and 33 in order. It can be seen that the number of flocculent particles that have been misclassified as mineral particles by Attention-CNN is significantly reduced, which indicates the focusing effect of attention mechanism.

(3) For spherical particles, Attention-CNN misclassifies them as mineral particles. CNN misclassifies them as fibers particles and mineral particles. SVM misclassifies them as the other three types of particles. Among them, the number of those misclassified as mineral particles is the largest. The number of spherical particles misclassified as mineral particles by Attention-CNN, CNN, and SVM is 13, 27, and 26, respectively, which also showed the focusing function of the attention mechanism.

(4) For the mineral particles, the three models have the problem of misclassifying them as other particles. Attention-CNN and CNN tend to misclassify them into spherical particles, and SVM tends to misclassify them as flocculent particles. However, the total number of misclassified particles using Attention$\mathrm{CNN}$ is the smallest, followed by CNN and SVM.

In summary, it can be obtained that Attention-CNN has a better classification performance than CNN and SVM, especially for fibrous particles, flocculent particles, and spherical particles. Compared with $\mathrm{CNN}$, Attention-CNN significantly reduces the number of particles misclassified after adding attention mechanism to $\mathrm{CNN}$, which indicates the focusing effect of the attention mechanism. Meanwhile, all of the three models have the problem of misclassifying fibrous particles, flocculent particles, and spherical particles as mineral particles, and the number of mineral particles misclassified is the largest compared with other particles, which shows that mineral particles have diverse morphological characteristics and are easily confused with other types of particulates.

In order to further evaluate the classification performance of Attention-CNN, four evaluation indexes, precision, recall, specificity, and $F 1$-score, are used and their calculation formulas are as follows:

$$
\begin{aligned}
\text { precision } & =\frac{\mathrm{TP}}{\mathrm{TP}+\mathrm{FP}}, \\
\text { recall } & =\frac{\mathrm{TP}}{\mathrm{TP}+\mathrm{FN}}, \\
\text { specificity } & =\frac{\mathrm{TN}}{\mathrm{TN}+\mathrm{FP}}, \\
F 1-\text { score } & =\frac{2 * \text { precision } * \text { recall }}{\text { precision }+ \text { recall }},
\end{aligned}
$$

where TP, FP, TN, and FN are the number of SEM images of PM2.5 classified as true positive, false positive, true negative, and false negative, respectively. F1-score is an evaluation index combining recall and precision, which is used to comprehensively reflect the overall results.

According to the test results of Attention-CNN, the above four evaluation indexes are obtained and compared with the results of CNN and SVM, as shown in Table 3.

From Table 3, it can be found that, in the identification and classification of fibrous particulates, spherical particulates, and flocculent particulates, the four evaluation indexes of Attention-CNN are superior to CNN and SVM. For the identification and classification of mineral particulates, recall and $F 1$-score of Attention-CNN are better than CNN and SVM, and precision and specificity are inferior to CNN but better than SVM. In general, the proposed Attention-CNN model is superior to CNN and 
Table 3: The four evaluation indexes of Attention-CNN, CNN, and SVM.

\begin{tabular}{|c|c|c|c|c|c|}
\hline Classification type & Models & Precision (\%) & Recall (\%) & Specificity (\%) & $F 1$-score $(\%)$ \\
\hline \multirow{3}{*}{ Fibrous } & Attention-CNN & 98.33 & 96.71 & 99.32 & 97.51 \\
\hline & $\mathrm{CNN}$ & 97.66 & 94.81 & 99.05 & 96.21 \\
\hline & SVM & 92.31 & 92.93 & 96.91 & 92.62 \\
\hline \multirow{3}{*}{ Flocculent } & Attention-CNN & 96.67 & 98.31 & 98.66 & 97.48 \\
\hline & $\mathrm{CNN}$ & 88.00 & 97.78 & 95.33 & 92.63 \\
\hline & SVM & 86.67 & 86.09 & 94.59 & 86.38 \\
\hline \multirow{3}{*}{ Spherical } & Attention-CNN & 94.17 & 89.74 & 98.39 & 91.90 \\
\hline & $\mathrm{CNN}$ & 87.00 & 92.82 & 96.51 & 89.81 \\
\hline & SVM & 86.10 & 91.87 & 96.27 & 88.89 \\
\hline \multirow{3}{*}{ Mineral } & Attention-CNN & 84.02 & 88.46 & 95.80 & 86.18 \\
\hline & $\mathrm{CNN}$ & 89.50 & 79.35 & 97.10 & 84.12 \\
\hline & SVM & 72.15 & 67.81 & 92.45 & 69.91 \\
\hline
\end{tabular}

Bold values indicate the best results of the four indexes in the three models.

SVM in the identification and classification of the atmospheric particulates SEM images.

\section{Conclusion}

In this paper, samples of PM2.5 are collected and their SEM images are obtained based on scanning electron microscopy. By analyzing the characteristics of SEM images of atmospheric particulates, they are divided into four types of typical particulates: fibrous particulates, flocculent particulates, spherical particulates, and mineral particulates. The Attention-CNN model for the identification and classification of atmospheric particles SEM images is established by integrating attention mechanism into CNN, which includes input layer, convolution layer, attention layer, full connection layer, and output layer. After Attention-CNN model is trained and tested by the SEM images database of atmospheric particulates, the classification results with high classification accuracy are obtained, which indicates the proposed Attention-CNN model is reasonable to identify and classify atmospheric particles SEM images.

In addition, the Attention-CNN model is compared with CNN and SVM models based on the same database. The results indicate Attention-CNN is superior to CNN and SVM in the classification of four types of atmospheric particles, which has higher classification accuracy and better evaluation indexes. Meanwhile, compared with CNN, Attention-CNN significantly reduces the number of particles misclassified after adding attention mechanism to $\mathrm{CNN}$, which indicates the focusing effect of the attention mechanism.

This study provides a reliable method for the automatic identification and classification of atmospheric particles. However, the morphological characteristics of atmospheric particulates are complex and diverse. This paper only focuses on the identification and classification of four types of particles and the types of particles can be expanded in the future. Moreover, how to introduce a better algorithm to improve the identification and classification accuracy of particulates with morphological diversity using $\mathrm{CNN}$ is also the content of further research.

\section{Data Availability}

The data used to support the findings of this study are available from the corresponding author upon request.

\section{Conflicts of Interest}

The authors declare that they have no conflicts of interest.

\section{Acknowledgments}

This work was supported by the National Natural Science Foundation of China Program (Nos. 61503224 and 61803234) and the Natural Science Foundation of Shandong Province of China Program (No. ZR2017MF048).

\section{References}

[1] R. J. Delfino, C. Sioutas, and S. Malik, "Potential role of ultrafine particles in associations between airborne particle mass and cardiovascular health," Environmental Health Perspectives, vol. 113, no. 8, pp. 934-946, 2005.

[2] J. Madriganl, I. Kloog, R. Goldberg, B. A. Coull, M. A. Mittleman, and J. Schwartz, "Long-term exposure to PM2.5 and incidence of acute myocardial infarction," Environmental Health Perspectives, vol. 121, no. 2, pp. 192-196, 2013.

[3] Y. Wang, I. Kloog, B. A. Coull, A. Kosheleva, A. Zanobetti, and J. D. Schwartz, "Estimating causal effects of long-term PM 2.5 exposure on mortality in New Jersey," Environmental Health Perspectives, vol. 124, no. 8, pp. 1182-1188, 2016.

[4] K. Adachi, S. H. Chung, and P. R. Buseck, "Shapes of soot aerosol particles and implications for their effects on climate," Journal of Geophysical Research, vol. 115, no. D15, Article ID D15206, 9 pages, 2010.

[5] È. Lecœur, C. Seigneur, C. Pagé, and L. Terray, "A statistical method to estimate PM2.5 concentrations from meteorology and its application to the effect of climate change," Journal of Geophysical Research: Atmospheres, vol. 119, no. 6, pp. 3537-3585, 2014.

[6] A. Genga, F. Baglivi, M. Siciliano et al., "SEM-EDS investigation on PM10 data collected in central Italy: principal component analysis and hierarchical cluster analysis," Chemistry Central Journal, vol. 6, no. S2, Article ID S3, 15 pages, 2012.

[7] V. S. Chithra and S. M. Shiva Nagendra, "Chemical and morphological characteristics of indoor and outdoor particulate matter in an urban environment," Atmospheric Environment, vol. 77, pp. 579-587, 2013.

[8] A. L. Dye, M. M. Rhead, and C. J. Trier, "The quantitative morphology of roadside and background urban aerosol in 
Plymouth, UK," Atmospheric Environment, vol. 34, no. 19, pp. 3139-3148, 2000.

[9] W. Akram, M. Madhuku, I. Ahmad, L. Xiaolin, G. Zhang, and L. Yan, "Morphology, microstructure and chemical composition of single inhalable particles in Shanghai, China," Environmental Monitoring and Assessment, vol. 186, no. 12, pp. 8587-8598, 2014.

[10] A. S. Pipal, A. Kulshrestha, and A. Taneja, "Characterization and morphological analysis of airborne PM2.5 and PM10 in Agra located in north central India," Atmospheric Environment, vol. 45, no. 21, pp. 3621-3630, 2011.

[11] Z. Zhao, S. Lv, Y. Zhang et al., "Characteristics and source apportionment of PM2.5 in Jiaxing, China," Environmental Science and Pollution Research, vol. 26, no. 8, pp. 7497-7511, 2019.

[12] W. Li and L. Shao, "Characterization of mineral particles in winter fog of Beijing analyzed by TEM and SEM," Environmental Monitoring and Assessment, vol. 161, no. 1-4, pp. 565-573, 2010.

[13] A. S. Pipal, R. Jan, P. G. Satsangi, S. Tiwari, and A. Taneja, "Study of surface morphology, elemental composition and origin of atmospheric aerosols (PM2.5 and PM10) over Agra, India," Aerosol and Air Quality Research, vol. 14, no. 6, pp. 1685-1700, 2014.

[14] B. Bahadar Zeb, K. Khan Alam, A. Armin Sorooshian, T. Blaschke, I. Ahmad, and I. Shahid, "On the morphology and composition of particulate matter in an urban environment," Aerosol and Air Quality Research, vol. 18, no. 6, pp. 1431-1447, 2018.

[15] C. Huang and P. Kuo, "A deep CNN-LSTM model for particulate matter (PM2.5) forecasting in Smart Cities," Sensors, vol. 18, no. 7, Article ID 2220, 22 pages, 2018.

[16] A. Chakma, B. Vizena, T. Cao, J. Lin, and J. Zhang, "Imagebased air quality analysis using deep convolutional neural network," in Proceedings of the IEEE International Conference on Image Processing, pp. 3949-3952, Beijing, China, September 2017.

[17] C. Wen, S. Liu, X. Yao et al., "A novel spatiotemporal convolutional long short-term neural network for air pollution prediction," Science of the Total Environment, vol. 654, pp. 1091-1099, 2019.

[18] P.-W. Soh, J.-W. Chang, and J.-W. Huang, "Adaptive deep learning-based air quality prediction model using the most relevant spatial-temporal relations," IEEE Access, vol. 6, pp. 38186-38199, 2018.

[19] J. Li, M. Jin, and H. Li, "Exploring spatial influence of remotely sensed PM2.5 concentration using a developed deep convolutional neural network model," International Journal of Environmental Research and Public Health, vol. 16, no. 3, Article ID 454, 11 pages, 2019.

[20] Q. Bo, W. Yang, N. Rija, Y. Xie, J. Feng, and J. Zhang, "Particle pollution estimation from images using convolutional neural network and weather features," in Proceedings of the 25th IEEE International Conference on Image Processing, pp. 3433-3437, Athens, Greece, October 2018.

[21] G. Ciocca, P. Napoletano, and R. Schettini, "CNN-based features for retrieval and classification of food images," Computer Vision and Image Understanding, vol. 176-177, pp. 70-77, 2018.

[22] J. Steinbrener, K. Posch, and R. Leitner, "Hyperspectral fruit and vegetable classification using convolutional neural networks," Computers and Electronics in Agriculture, vol. 162, pp. 364-372, 2019.
[23] S. Deepak and P. M. Ameer, "Brain tumor classification using deep CNN features via transfer learning," Computers in Biology and Medicine, vol. 111, Article ID 103345, 7 pages, 2019.

[24] Z. Wu, S. Zhao, Y. Peng et al., "Studies on different CNN algorithms for face skin disease classification based on clinical images," IEEE Access, vol. 7, pp. 66505-66511, 2019.

[25] Y. Zhou, J. Xu, Q. Liu et al., "A radiomics approach with CNN for shear-wave elastography breast tumor classification," IEEE Transactions on Biomedical Engineering, vol. 65, no. 9, pp. 1935-1942, 2018.

[26] R. Jain, N. Jain, A. Aggarwal, and D. J. Hemanth, "Convolutional neural network based Alzheimer's disease classification from magnetic resonance brain images," Cognitive Systems Research, vol. 57, pp. 147-159, 2019.

[27] M. Paoletti, J. Haut, J. Plaza, and A. Plaza, "Deep\&dense convolutional neural network for hyperspectral image classification," Remote Sensing, vol. 10, no. 9, Article ID 1454, 28 pages, 2018.

[28] Y. Li, W. Xie, and H. Li, "Hyperspectral image reconstruction by deep convolutional neural network for classification," Pattern Recognition, vol. 63, pp. 371-383, 2017.

[29] W. Hu, Y. Huang, L. Wei, F. Zhang, and H. Li, "Deep convolutional neural networks for hyperspectral image classification," Journal of Sensors, vol. 2015, Article ID 258619 , 12 pages, 2015.

[30] M. E. Paoletti, J. M. Haut, J. Plaza, and A. Plaza, "A new deep convolutional neural network for fast hyperspectral image classification," ISPRS Journal of Photogrammetry and Remote Sensing, vol. 145, pp. 120-147, 2018.

[31] S. Li, X. Zhu, and J. Bao, "Hierarchical multi-scale convolutional neural networks for Hyperspectral Image Classification," Sensors, vol. 19, no. 7, Article ID 1714, 20 pages, 2019.

[32] N. Ouyang, T. Zhu, and L. Lin, "Convolutional neural network trained by joint loss for hyperspectral image classification," IEEE Geoscience and Remote Sensing Letters, vol. 16, no. 3, pp. 457-461, 2019.

[33] J. Ma, K. Li, Y. Han, and J. Yang, "Image-based air pollution estimation using hybrid convolutional neural network," in Proceedings of the 24th International Conference on Pattern Recognition, pp. 471-476, Beijing, China, August 2018.

[34] L. Li, M. Xu, H. Liu et al., "A large-scale database and a CNN model for attention-based glaucoma detection," IEEE Transactions on Medical Imaging, vol. 39, no. 2, pp. 413-424, 2020.

[35] R. Karthik, M. Hariharan, S. Anand, P. Mathikshara, A. Johnson, and R. Menaka, "Attention embedded residual CNN for disease detection in tomato leaves," Applied Soft Computing, vol. 86, Article ID 105933, 12 pages, 2020.

[36] S. Li, G. Xie, J. Ren, L. Guo, Y. Yang, and X. Xu, "Urban PM2.5 concentration prediction via attention-based CNN-LSTM," Applied Sciences-Basel, vol. 10, no. 6, Article ID 1953, 17 pages, 2020.

[37] Y. Song, F. He, and X. Zhang, "To identify tree species with highly similar leaves based on a novel attention mechanism for CNN," IEEE Access, vol. 7, pp. 163277-163286, 2019.

[38] L. Niu, C. Yin, X. Liu, M. Zhao, and X. Cheng, "An image segmentation method of atmosphere particulates based on iteration diameter circle detection," China Science Paper, vol. 13, no. 20, pp. 2330-2336, 2018.

[39] H. Xue, G. Liu, H. Zhang, R. Hu, and X. Wang, "Elemental composition, morphology and sources of fine particulates (PM2.5) in Hefei city, China," Aerosol and Air Quality Research, vol. 19, no. 8, pp. 1688-1696, 2019. 
[40] D. Bahdanau, K. Cho, and Y. Bengio, "Neural machine translation by jointly learning to align and translate," Lecture Notes in Computer Science, Springer, Berlin, Germany, 2016.

[41] S. Wang, J. Na, and Y. Xing, "Adaptive optimal parameter estimation and control of servo mechanisms: theory and experiments," IEEE Transactions on Industrial Electronics, 2020.

[42] R. V. Kumar Reddy, B. Srinivasa Rao, and K. P. Raju, "Handwritten Hindi digits recognition using convolutional neural network with RMSprop optimization," in Proceedings of the 2018 Second International Conference on Intelligent Computing and Control Systems, pp. 45-51, Madurai, India, June 2018

[43] S. Wang, L. Tao, Q. Chen, J. Na, and X. Ren, "USDE-based sliding mode control for servo mechanisms with unknown system dynamics," IEEE/ASME Transactions on Mechatronics, vol. 25, no. 2, pp. 1056-1066, 2020.

[44] S. Wang and J. Na, "Parameter estimation and adaptive control for servo mechanisms with friction compensation," IEEE Transactions on Industrial Informatics, vol. 16, no. 11, pp. 6816-6825, 2020.

[45] D. P. Kingma and J. Ba, "Adam: a method for stochastic optimization," Lecture Notes in Computer Science, Springer, Berlin, Germany, 2017. 